\title{
Chronic effects of an invasive species on an animal community
}

\author{
J. Sean Doody, ${ }^{1,2,5}$ David Rhind, ${ }^{3}$ Brian Green, ${ }^{4}$ Christina Castellano, ${ }^{4}$ Colin McHenry, ${ }^{2}$ and Simon Clulow ${ }^{2}$ \\ ${ }^{1}$ Department of Biological Sciences, Southeastern Louisiana University, Hammond, Louisiana 70402 USA \\ ${ }^{2}$ School of Environmental and Life Sciences, University of Newcastle, Callaghan, New South Wales 2308 Australia \\ ${ }^{3}$ School of Biological Sciences, Monash University, Clayton, Victoria 3800 Australia \\ ${ }^{4}$ Institute for Applied Ecology, University of Canberra, Canberra, Australian Capital Territory 2601 Australia
}

Abstract. Invasive species can trigger trophic cascades in animal communities, but published cases involving their removal of top predators are extremely rare. An exception is the invasive cane toad (Rhinella marina) in Australia, which has caused severe population declines in monitor lizards, triggering trophic cascades that facilitated dramatic and sometimes unexpected increases in several prey of the predators, including smaller lizards, snakes, turtles, crocodiles, and birds. Persistence of isolated populations of these predators with a decadeslong sympatry with toads suggests the possibility of recovery, but alternative explanations are possible. Confirming predator recovery requires longer-term study of populations with both baseline and immediate post-invasion densities. Previously, we quantified short-term impacts of invasive cane toads on animal communities over seven years at two sites in tropical Australia. Herein, we test the hypothesis that predators have begun to recover by repeating the study $12 \mathrm{yr}$ after the initial toad invasion. The three predatory lizards that experienced $71-97 \%$ declines in the short-term study showed no sign of recovery, and indeed a worse fate: two of the three species were no longer detectable in $630 \mathrm{~km}$ of river surveys, suggesting local extirpation. Two mesopredators that had increased markedly in the short term due to these predator losses showed diverse responses in the medium term; a small lizard species increased by $\sim 500 \%$, while populations of a snake species showed little change. Our results indicate a system still in ecological turmoil, having not yet reached a "new equilibrium" more than a decade after the initial invasion; predator losses due to this toxic invasive species, and thus downstream effects, were not transient. Given that cane toads have proven too prolific to eradicate or control, we suggest that recovery of impacted predators must occur unassisted by evolutionary means: dispersal into extinction sites from surviving populations with alleles for toxin resistance or toad avoidance. Evolution and subsequent dispersal may be the only solution for a number of species or communities affected by invasive species for which control is either prohibitively expensive, or not possible.

Key words: cane toad; Crocodylus; Dendrelaphis; Lophognathus; mesopredator; monitor lizards; recovery; Rhinella marina; top predator; trophic cascades; Varanus.

\section{INTRODUCTION}

The removal of top predators can have dramatic and widespread effects on communities and ecosystems. Evidence is mounting for predator losses triggering trophic cascades across ecosystems worldwide; these cascades influence a wide array of components and processes including biodiversity, water, wildfire, soils, disease, and atmosphere (Estes et al. 2011). Given the seriousness of predator losses and their downstream effects, a priority for managers is to find ways to restore top predators to ecosystems.

In theory, there are two ways in which communities or ecosystems could return to their pre-perturbation condition. First, predator densities could be restored via management actions; if their chief threat(s) is removed,

Manuscript received 7 October 2016; revised 19 February 2017; accepted 5 April 2017. Corresponding Editor: Brian D. Todd

${ }^{5}$ E-mail: jseandoody@gmail.com predators could be successfully reintroduced into the ecosystem. For example, the reintroduction of grey wolves (Canis lupus) to Yellowstone National Park, through its effects on elk (Cervus elaphus), has apparently caused the recovery of riparian plant communities (Ripple and Beschta 2007, Beschta and Ripple 2013). A second possibility is the unassisted recovery of predator populations over time via evolutionary mechanisms; evolution can occur within timeframes that are relevant to most conservation issues (Stockwell et al. 2003). Long-term studies demonstrating predator recovery following a perturbation via evolutionary means are generally lacking, but theory suggests that they occur.

A major perturbation that can remove top predators is a species invasion. Invasive species can trigger trophic cascades in communities, but cases involving invasive species removing top predators are rare (Rodriguez 2006, White et al. 2006, Doody et al. 2015). An exception is the invasive cane toad (Rhinella marina) in Australia, which has caused severe population declines in animals including top predators (Letnic et al. 2008, 
Doody et al. 2009, 2014, Ujvari and Madsen 2009, Woinarski et al. 2010). Predators, which succumb to the skin toxins of toads while attempting to ingest them, are evolutionarily naive to the toxins of the toads because true toads are not native to Australia (Lever 2001). In a compelling example, three species of monitor lizards suffered severe population-level declines (Doody et al. 2006, 2009), causing them to attain conservation listing as "vulnerable" at the state level (Ward et al. 2006, 2012, Ward 2012). Moreover, the loss of these predators triggered trophic cascades that resulted in dramatic and sometimes unexpected increases in several prey of the predators including smaller lizards, snakes, turtles, crocodiles, and birds (Doody et al. 2006, 2009, 2013, 2015, Webb and Manolis 2010).

Cane toads, which have now invaded more than 50 countries worldwide (Lever 2001), have proven too prolific to eradicate or control (Shine and Doody 2011), precluding the option of removing the threat and reintroducing the predators. However, could there be unassisted recovery of predator populations over time? For example, if survivors reflect heritable resistance to the toxins, those genes could become fixed in small populations, resulting in recovery through successive generations, and subsequent dispersal into extinction sites. It is also plausible that prey preference in these predators is heritable, and thus the survivors simply prefer not to eat toads, a preference that could become fixed in a growing, recovering population. Alternatively, survivors may thwart death by getting a sublethal dose of toxins and subsequently learn from the encounter, thus avoiding toads in future encounters (Ward-Fear et al. 2016). However, in the latter case, the survivors' progeny would be expected to suffer the same high rate of mortality as their parents, because these reptilian predators do not interact with their offspring and are not likely gregarious enough to benefit from social learning (Pianka and King 2004).

What is the evidence that monitor lizards might eventually gain resistance to, or avoidance of, toxic toads? And what is the evidence for recovery of the lizards where they have coexisted with cane toads for long periods of time? Over the longer term, monitor lizards from Asia and Africa have coexisted with toads for millions of years (Ujvari et al. 2013, 2015), and resistance to toad toxins in some species is confirmed by toads in their diet (Losos and Green 1988). Toad toxins target the $\mathrm{H} 1-\mathrm{H} 2$ domain of the $\alpha_{1}$ subunit of the sodium-potassium-ATPase enzyme, and, compared to the Asian-African monitor lizards, the Australian monitor lizards show four-base-pair substitutions, resulting in alterations of three of the 12 amino acids in the H1-H2 domain (Ujvari et al. 2013, 2015). Tellingly, human embryonic kidney cells transfected with $\mathrm{H} 1-\mathrm{H} 2$ domain cells from Australian monitor lizards exhibited a 3000 -fold decrease in resistance to toad toxins (Bufalin) compared to H1-H2 domain cells from AsianAfrican monitor lizards (Ujvari et al. 2013, 2015).

Thus, a genotype-phenotype linkage for toxin resistance in monitor lizards has been established, suggesting that (some) surviving lizards in Australian populations may be genetically resistant to toad toxins, which could eventually lead to predator recovery in populations that are sympatric with toads. Unfortunately, there has been no direct evidence that post-toad recovery has occurred in populations of these predators. For example, although Varanus panoptes persists in pockets in several locations where it has been sympatric with toads for up to $75 \mathrm{yr}$ (Shine 2010; Doody et al., unpublished data), no baselines exist to confirm that recovery has occurred. It is possible that these populations existed at high densities prior to toad arrival and impact, or, due to toxin resistance, toad avoidance or some unknown reason, were never severely impacted by toads in the first place. Baseline population abundance data are necessary to determine if post-toad recovery has occurred, and thus determine the best strategies for mitigating impacts.

Although short-term effects of invasive species are important to understand because they can have serious ecological or economic consequences, understanding ecological and evolutionary processes that modulate the effects of invasive species requires long-term study (Strayer et al. 2006). Unfortunately, most studies of the effects of invasive species have been brief and lack a temporal context (Strayer et al. 2006). Previously, we quantified short-term impacts of the invasive cane toad on an animal community at two sites in tropical Australia over seven years (Doody et al. 2009). Toads arrived in the third year of the study, causing severe population-level declines (71-97\%) in three species of monitor lizards by the fourth and fifth years, followed by precipitous declines to very low numbers in years six and seven. This decline in turn caused increases in several species of mesopredators (Doody et al. 2009, 2013, 2015). We recently repeated our study to determine if populations of monitor lizards have begun to recover $12 \mathrm{yr}$ after the initial toad invasion (although generation time is unknown in nature, in captivity these predators can reach maturity in one year [Paden 2008]). We tested the hypothesis that predator populations have begun to recover, and thus, mesopredators have begun to decrease. To achieve this, we determined the relative density of predators: three species of monitor lizards (Varanus panoptes, V. mertensi, V. mitchelli). two species of crocodiles (Crocodylus johnstoni and C. porosus), and two mesopredaors: Gilbert's dragons (Lophognathus gilberti), and common tree snakes (Dendrelaphis punctulata). We compared the above densities of predator and prey to those in our previous study to determine if short-term effects were transient in the medium term. We provide additional context by discussing implications for control vs. unassisted recovery via evolutionary means in toads and in other invasive species.

\section{Methods}

The yellow-spotted monitor ( $V$. panoptes), Mitchell's monitor ( $V$. mitchelli), and Merten's water monitor 
( $V$. mertensi) are medium- to large-sized carnivorous lizards endemic to northern Australia. They are generalist predators that are known to ingest small- to mediumsized cane toads (Rhinella marina; Catling et al. 1999, Doody et al. 2006, Griffiths and McKay 2007) and they readily suffer population-level declines (Doody et al. 2009, 2014, 2015, Ujvari and Madsen 2009). The larger $V$. panoptes is primarily terrestrial, whereas $V$. mitchelli is also arboreal and semi-aquatic, and $V$. mertensi is highly aquatic (Cogger 2000). All three lizards can be observed foraging and basking on fallen logs, pandanus trees, and riverbanks.

The freshwater crocodile (Crocodylus johnstoni) is also a northern Australian endemic, inhabiting freshwater wetlands and occasionally tidal zones of river systems (Webb and Manolis 2010). Crocodylus johnstoni consumes most animals and cane toads have been extracted from its stomach contents (Letnic and Ward 2005), and populations declined in at least one system (Letnic et al. 2008). Although primarily nocturnal, this species can be observed basking throughout the day, especially during the winter dry season (Seebacher and Grigg 1997). The saltwater crocodile (C. porosus) inhabits a variety of saltwater habitats and lower stretches of rivers (Webb and Manolis 2010). It consumes virtually all animals, and basks during the day in the dry season (Webb and Manolis 2010).

Gilbert's dragon (Lophognathus gilbert) is a small agamid lizard that is widespread throughout northern Australia (Cogger 2000). It is mainly arboreal (Cogger 2000), but can be observed foraging and scurrying on riverbanks throughout the day (S. Doody, personal observation). It is not known to consume toads, but is a prey item of $V$. panoptes (Doody et al. 2012a), and it experienced toad-induced mesopredator release at our study sites (Doody et al. 2009). The common tree snake (Dendrelaphis punctulata) is a medium-sized snake of northern and eastern Australia (Cogger 2000). It is a diurnal forager that preys upon frogs, lizards, and insects (Cogger 2000). It may consume smaller cane toads ((Covacevich and Archer 1975), but see (Phillips et al. 2003)), is a prey item of $V$. panoptes (Doody et al. $2012 b$ ), and it experienced toad-induced mesopredator release at our study sites (Doody et al. 2013).

To determine the relative abundance of predators and prey, we conducted five all-day, $70 \mathrm{~km}$-long surveys at two sites along the Daly River during 19-28 May 2015. The upper Daly site was near Oolloo Crossing $\left(14^{\circ} 00^{\prime} 18^{\prime \prime} \mathrm{S}, 131^{\circ} 14^{\prime} 25^{\prime \prime} \mathrm{E}\right.$ to $\left.13^{\circ} 47^{\prime} 17^{\prime \prime} \mathrm{S}, 131^{\circ} 17^{\prime} 49^{\prime \prime} \mathrm{E}\right)$ and the lower Daly site was near Daly River Township (1346 $29^{\prime \prime} \mathrm{S}, 130^{\circ} 43^{\prime} 16^{\prime \prime}$ E to $\left.13^{\circ} 56^{\prime} 51^{\prime \prime} \mathrm{S}, 130^{\circ} 51^{\prime} 27^{\prime \prime} \mathrm{E}\right)$. The two sites are $\sim 30 \mathrm{~km}$ apart, and are similar in habitat types and animal communities; however, deeper water in the lower Daly site favors higher numbers of crocodiles. Our methods strictly followed those in the previous study (Doody et al. 2009). Three observers in a flat-bottomed boat travelling at $\sim 10 \mathrm{~km} / \mathrm{h}$ scanned microhabitats (e.g., riverbanks, trees, and $\operatorname{logs}$ ) along one side of the river for
$35 \mathrm{~km}$, followed by a return search along the opposite side of the river for the same $35-\mathrm{km}$ section. Surveys began at 08:30 and finished at 17:30. The boat was maintained at a distance $7-10 \mathrm{~m}$ from the shoreline throughout the surveys, which were performed on sunny days with little to no cloud cover. The species and time of day were recorded when a study animal was observed. Air and water temperatures were also recorded at 08:30, 10:30, 15:30, and 17:30 during each survey.

\section{Statistical analyses}

Differences in counts for each of the study species were investigated for the three toad-invasion phases: pre-toad invasion (2001 - 2003 at upper Daly site; 2003 at lower Daly site), short-term post-invasion (the initial year of invasion plus three years, 2004-2007 at both sites [the results of the pre-toad and short-term post-invasion study were previously reported in Doody et al. (2009)]), and medium-term post-invasion (2015 at both sites, $12 \mathrm{yr}$ post-invasion; the focus of the current study).

For each data set, a Generalized Linear Mixed Model (GLMM) with Poisson distribution and log link function was performed in SAS (version 9.4; SAS Institute, Cary, North Carolina, USA) using the glimmix procedure, with fixed effects for toad invasion stage, site, the interaction between the two, and mean air and water temperature. A random effect of survey year was added to account for year to year variability that was not explained by either toad invasion stage or temperature. For Varanus panoptes and $V$. mitchelli, where a zero count in all surveys performed in the medium-term invasion stage was recorded, a single record for each species was added to the data set for one survey period in order for the models to be able to converge. This approach would theoretically lead to a slight underestimation of the significance and magnitude of decline for those species in the medium-term, which is thus a conservative approach. In cases where toad invasion stage was found to be a significant driver of animal numbers, differences in least squares means were used to assess whether a significant change in counts had occurred specifically between the short-term and medium-term invasion stages. In cases where there was a significant effect of site or an interaction between site and invasion stage (indicating that the increase or decrease in animal numbers between the sites differed in pattern), differences in least squares means were investigated for the two sites independently to comment on the patterns of change. All reported means for invasion stage are fitted model means except in the cases of Varanus panoptes and $V$. mitchelli in the medium-term invasion stage where a false record was added; in these cases, the reported mean remained as zero. In cases where animal counts decreased or increased significantly due to toad invasion stage, the magnitude of the change was calculated as a percentage by dividing the mean survey count of the latter invasion stage by the mean count of the former invasion stage, and multiplying by 100 . 


\section{RESULTS}

We found no evidence of recovery of the three species of predatory lizards that had declined by $71-97 \%$ in the shorter-term study; rather, two of the three species ( $V$. panoptes and $V$. mitchelli) declined further to the point that they could no longer be detected in the current study, indicating possible local extirpation
(Fig. 1). For $V$. panoptes, there was a significant effect of toad invasion stage $\left(F_{2,56}=9.46, P<0.001\right)$ with declines across all three stages (fitted model means decreased from 12.90 to 1.65 to 0 ; pre-toad to short-term postinvasion to medium-term post-invasion, respectively; Fig. 1a, b). The decline was consistent between sites and significant between the short- and medium-term postinvasion $(t=1.96, \mathrm{df}=56, P=0.05)$. For $V$. mitchelli,
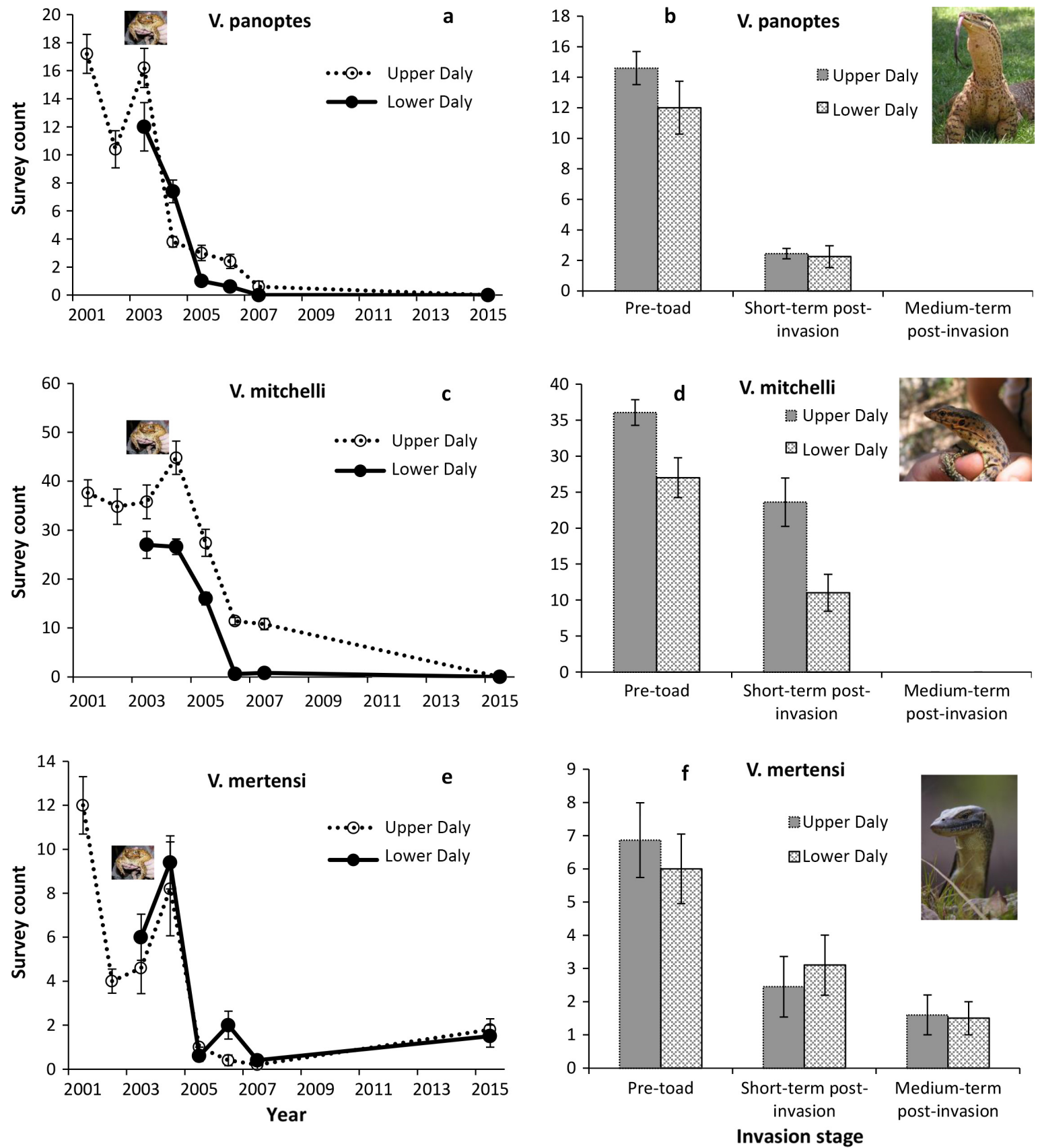

FIG. 1. Counts per survey of predatory monitor lizards (Varanus panoptes, V. mertensi, V. mitchelli; a, c, e) between 2001 and 2015, and (b, d, f) by toad invasion stage along the Upper and Lower Daly River, Australia. The toad photograph denotes the timing of toad arrival into the system. Values are mean \pm SE. [Color figure can be viewed at wileyonlinelibrary.com] 
there was a significant interaction between site and toad invasion stage $\left(F_{2,56}=5.43, P<0.01\right)$ due to slight differences in the magnitude and speed to decline from pretoad to short-term post-toad invasion (likely due to the small, unexplained spike in 2004 at upper Daly; Fig. 1c). Declines occurred, however, across all three invasion stages at both sites, with fitted model means of 29.18 , 8.39, and 0 at the lower Daly, and 36.28, 5.88, and 0 at the upper Daly pre-toad, short-term post-invasion, medium-term post-invasion, respectively (Fig. 1c, d). The declines between the short- and medium-term post-invasion were significant at both sites $(t=2.68$, df $=56$, $P<0.01$ lower Daly; $t=3.48, \mathrm{df}=56, P=0.001$ upper Daly). Despite a significant decrease upon the arrival of toads (see Doody et al. 2009), the third species (V. mertensi) showed no change between the short-term and medium-term studies (Fig. 1e, f), remaining at a similar low abundance. Fitted model means decreased from 6.28 pre-toad invasion to 1.35 in the short-term post-invasion but leveled off at 1.39 in the medium-term post-invasion. There was also a significant positive effect of mean water
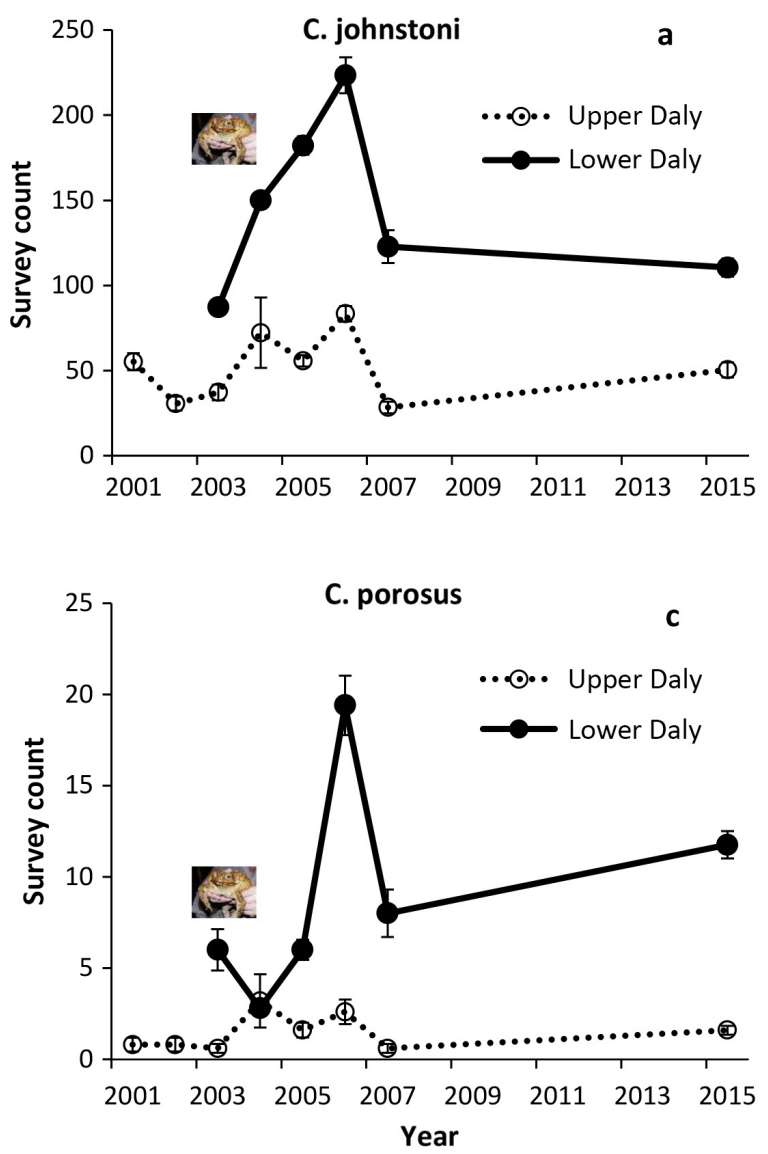

FIG. 2. Mean counts per survey of crocodiles (Crocodylus johnstoni and C. porosus; a, c) between 2001 and 2015 and (b, d) by toad invasion stage. The toad photograph denotes the timing of toad arrival into the system. Values are mean \pm SE. [Color figure can be viewed at wileyonlinelibrary.com]
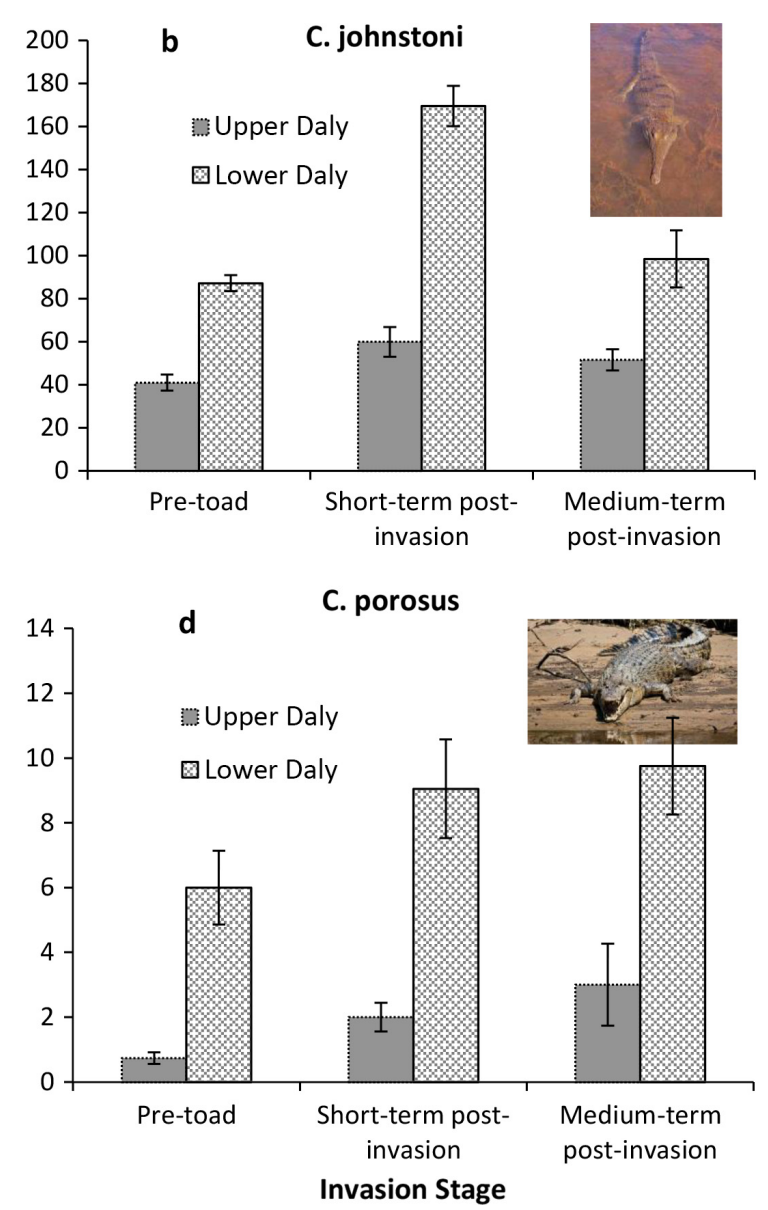

temperature on counts of both of the water monitors, $V$. mitchelli $\left(F_{1,56}=4.51, \quad P=0.04\right)$ and $V$. mertensi $\left(F_{1,56}=6.82, P=0.01\right)$.

The other species of top predator in our study system (crocodiles), which did not decline with toad arrival in the shorter-term study (Doody et al. 2009), showed no major changes between the short- and medium-term post-invasion (Fig. 2). For freshwater crocodiles (C. johnstoni) there was a significant interaction between site and toad invasion stage $\left(F_{2,56}=4.55, P<0.01\right)$ due to a temporary spike in counts in the short-term postinvasion at both sites (fitted model means $=81.17$ pretoad and 147.51 short-term post-toad invasion at lower Daly, $t=3.21$, df $=56, P<0.01$ lower Daly; and 38.83 pre-toad to 66.59 short-term post-toad invasion at upper Daly, $t=3.04$, df $=56, P<0.01$; Fig. 2a, b). The spike in counts at the lower Daly decreased significantly in the medium-term post-invasion (fitted model mean $=87.66$; $t=2.05, \mathrm{df}=56, P<0.05)$ at which time counts were not significantly different to pre-toad numbers $(t=0.28$, $\mathrm{df}=56, P=0.78)$. Mean counts also decreased at the 
upper Daly site in the medium-term post-invasion (fitted model mean $=51.23$ ) but were not significantly different from either the short-term post-invasion or pre-toad invasion stages. For saltwater crocodiles (C. porosus), there was an effect of site on counts indicating that the numbers of crocodiles at the two sites differed ( $F_{1,56}=49.06, P<0.001$; Fig. $2 \mathrm{c}$, d) but no effect of toad invasion stage or an interaction between the two was found. Fitted model means were 5.85, 6.66, and 8.24 at the lower Daly and $0.68,2.27$, and 1.65 at the upper Daly (pre-toad, short-term post-invasion, medium-term post-invasion, respectively). There was also a significant inverse effect of mean water temperature on mean counts of both crocodile species $\left(F_{1,56}=68.87\right.$, $P<0.001$ for $C$. johnstoni and $F_{1,56}=8.69, P<0.01$ for $C$. porosus) as well as an inverse effect of air temperature on mean counts of $C$. johnstoni $\left(\mathrm{F}_{1,56}=6.87, P=0.01\right)$.

The two mesopredators that had increased in the shorter-term study due to predator losses showed diverse responses in the medium term (Fig. 3). Gilbert's dragons ( $A$. gilberti) increased dramatically between the shorter-term and current studies (Fig. 3a, b). There was a significant effect of toad invasion stage $\left(F_{2,56}=6.38\right.$,
$P<0.01)$ and site $\left(F_{1,56}=10.27, P<0.01\right)$ on mean counts, but no interaction between the two. Counts increased across all three invasion stages with fitted model means of 4.06, 14.70, and 87.44 at the lower Daly and 6.30, 17.71, and 117.82 at the upper Daly (pre-toad, short-term post-invasion, medium-term post-invasion, respectively; Fig. 3a, b). This represents increases in mean counts of Gilbert's dragons of $495 \%$ at the lower Daly and $565 \%$ at the upper Daly from the short to the medium-terms, on top of the 262\% and 181\% already observed at the lower Daly and upper Daly sites respectively from pre-toad invasion to short-term post-invasion (or total increases in mean counts of $2054 \%$ and $1770 \%$ from pre-toad to the medium-term post-invasion at the lower and upper Daly, respectively). In contrast, tree snakes (D. punctulata), which increased in the shorter-term study (Doody et al. 2013), remained at similar abundances between the short and medium-term studies (Fig. 3c, d). While there was a significant site by toad invasion stage interaction $\left(F_{2,56}=10.44\right.$, $P<0.001)$ due to the differences in the pattern of increase/decrease in mean counts in the years 2007 and 2015 (Fig. 3c, d), there was no significant increase in
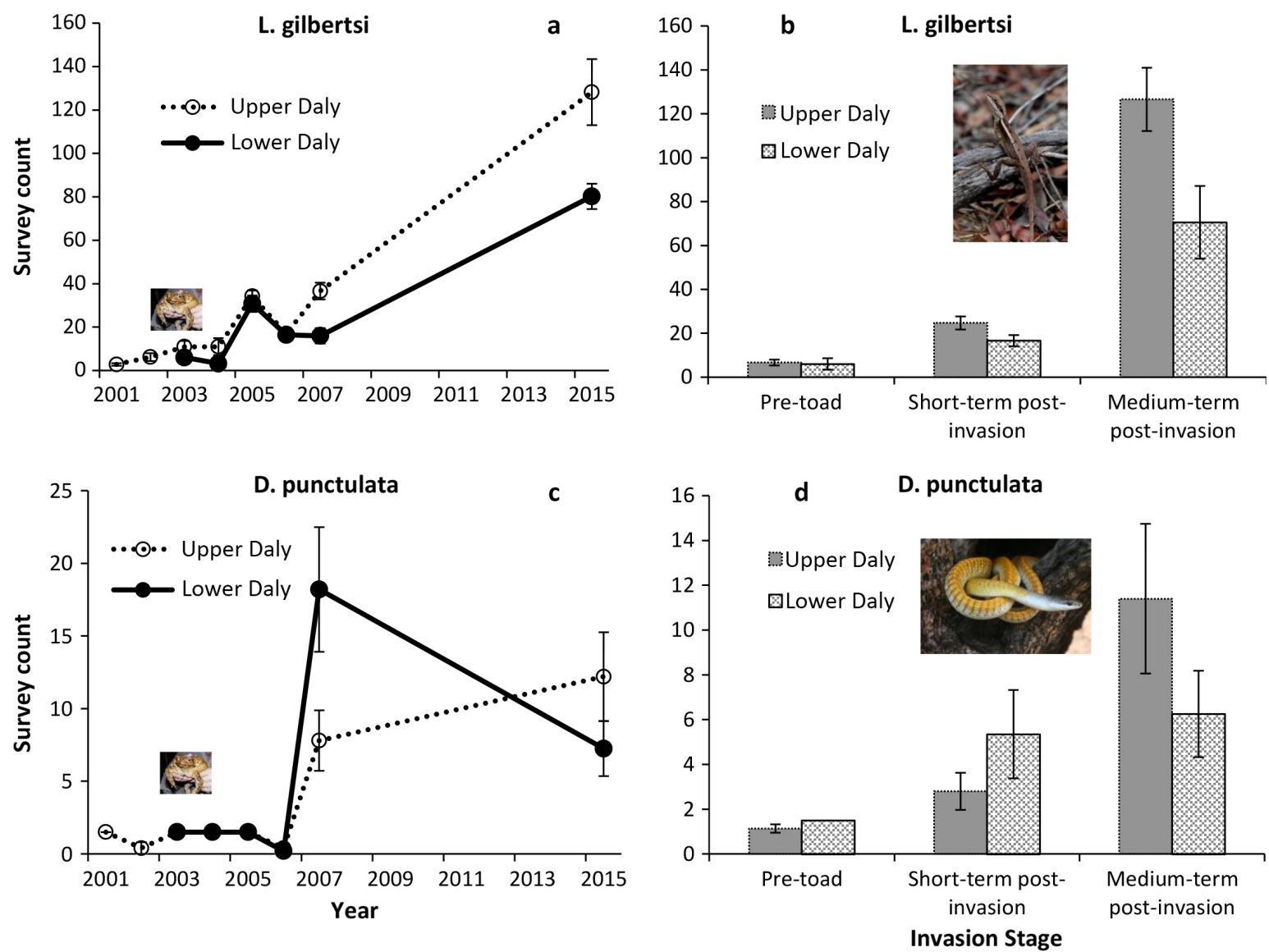

FIG. 3. Mean counts per survey of mesopredators (Gilbert's dragons [Lophognathus gilberti] and common tree snakes [Dendrelaphis punctulata]; a, c) between 2001 and 2015 and (b, d) by toad invasion stage. The toad photograph denotes the timing of toad arrival into the system. Values are mean $\pm \mathrm{SE}$. [Color figure can be viewed at wileyonlinelibrary.com] 
counts between the short-term and medium-term postinvasion stages at either site (although a small increase at the upper Daly site was bordering on significant; $t=-1.95, \mathrm{df}=56, P=0.06$ ). For both mesopredators, there were significant effects of air $\left(F_{1,56}=9.17\right.$, $P<0.01$ for $A$. gilberti and $F_{1,56}=8.13, P<0.01$ for D. punctulata $)$ and water $\left(F_{1,56}=15.30, P<0.001\right.$ for A. gilberti and $F_{1,56}=11.75, P<0.01$ for $D$. punctulata) temperature. However, these effects were positive for A. gilberti and inverse for $D$. punctulata.

\section{DisCUSSION}

Invasive cane toads are a serious conservation problem due to their virtual removal of top predators from animal communities; for example, we showed that toads decimated populations of three Australian predator (lizard) species over the shorter term (4 yr post-invasion; Doody et al. 2009). Reintroduction of these predators would not be a viable strategy, because the chief threat to their populations, the cane toad, has proven too prolific to eradicate or control (Shine and Doody 2011). Thus, toad-impacted predators must recover on their own via evolutionary means and subsequent dispersal. However, our current study demonstrated that populations have not begun to recover over the medium term (12 yr post-invasion). Indeed, two of the three predatory lizard species persisting at low abundances in the shorter-term study declined further to the point of not being detectable in the current, medium-term study, indicating possible extirpations from their communities. The third predatory lizard species remained at low abundances between the shorter-term and medium-term studies, while crocodiles remained unaffected at the population level. Two mesopredators that had increased in the shorter-term due to the above predator losses showed diverse responses in the medium term. Gilbert's dragons, which had already increased dramatically in numbers in the short term, increased dramatically (and unexpectedly) again between the shorter and medium terms, leading to a staggering $2000 \%$ increase in numbers of an already common lizard. In contrast, common tree snakes, however, leveled off between the short- and medium-term studies. Collectively, our study indicates that (1) recovery of predators to an invasive species has not begun; in stark contrast, two species may have been extirpated; (2) downstream cascading effects are thus still evident over the medium-term but are variable among species; and thus (3) cane toads appear to be having a more lasting and far-reaching impact, in terms of trophic levels affected, than previously thought; and (4) accurate assessment of the effects of invasive species on biological communities can require longer-term study. Given that cane toads have proven too prolific to eradicate or control, we suggest that recovery of impacted predators must occur unassisted by evolutionary means.

The coexistence of monitor lizards with toxic toads in Asia and Africa (Doody et al. 2013), along with the inclusion of toads in the diet in some monitor species in those areas (Losos and Green 1988) provides the potential for evolutionary responses that could facilitate unassisted re-colonization. Persistence of isolated populations of these predators with a decades-long sympatry with toads in Australia also suggests the possibility of recovery (Shine 2010), but alternative explanations are possible (e.g., remnant populations were never impacted in the first place). The only way to confirm recovery is through longer-term study of populations with both baseline densities and immediate post-invasion densities. Because generation time in these lizard predators can be as rapid as one year (in captivity; Paden 2008), theoretically enough time has elapsed for surviving individuals to reproduce, build up numbers of toad-avoiding or toxin-resistant individuals, and thus begin population recovery.

However, the hypothesis that predator populations that were previously severely impacted by invasive cane toads have begun to recover, was not supported by the present study. In contrast, we could no longer detect $V$. panoptes or $V$. mitchelli in $630 \mathrm{~km}$ of surveys (one $70-\mathrm{km}$ survey was cancelled due to logistical problems; Fig. 1). Prior to the toad invasion we would typically count $\sim 150$ and $\sim 350$ of these predators annually, respectively, and during the last post-invasion year in the short-term study we were counting 1-12 individuals of these predators. Our failure to detect these two species in 216 person hours of surveys over $630 \mathrm{~km}$ in the medium-term (2015) study suggests that their populations have incurred local to regional extinctions along the Daly River. This is supported by anecdotes in other areas of the Northern Territory that indicate the disappearance of these species after the arrival of cane toads (Doody et al., unpublished data). However, in certain areas, especially coastal ecosystems, isolated populations of both species remain after $\sim 10 \mathrm{yr}$ post-invasion (Doody et al., unpublished manuscript). The lack of population recovery of these two predators in the current study could be explained by (1) local or regional extinctions; (2) populations that have not yet begun to recover to a detectable level due to very small population sizes and/or insufficient number of generations since the toad invasion; or (3) the lack of heritable resistance to toxins in the populations. In the latter case, recovery may require secondary colonization from surviving predator populations with the genetic makeup for toxin resistance (Ujvari et al. 2015).

Cane toads in Australia have frustrated all attempts to eradicate or control them. A biocontrol program was terminated prior to the development of a control pathogen (Shanmuganathan et al. 2010), and various attempts to control toads by hand or other means have failed to noticeably reduce numbers in nature (Shine and Doody 2011). The chief threat to the lizard predators thus remains, precluding the option of human-assisted reintroduction. Although recent research proposes that these lizards could be "taught" to avoid toads through aversion learning (Ward-Fear et al. 2016), cultural inheritance is 
highly unlikely because parental abandonment of eggs precludes teaching offspring to avoid toads in these reptiles. Thus, if $90 \%$ of lizard parents die while attempting to ingest cane toads, $90 \%$ of their offspring will similarly perish. Thus, aversion learning would provide a conservation tool that would only provide protection for a (small) group of one generation of lizards. Rather, these predators are faced with recolonizing on their own, presumably after they have either evolved behavioral avoidance of, or toxin resistance to, cane toads.

Our study also demonstrated a diversity of mediumterm responses of predators and prey to the cane toad invasion. Unlike the other two monitor lizard species, counts of $V$. mertensi did not decline further, but remained low between the shorter-term and current studies (Fig. 1). The mechanism accounting for the difference in this response compared to those of $V$. panoptes and $V$. mitchelli is unknown. Crocodile counts were also similar between shorter- and longer-term studies, and seemed overall to show negligible effects from cane toads (Fig. 2; see also Doody et al. 2009). Long-term monitoring of freshwater crocodiles in the same system prompted Fukuda et al. (2015) to invoke toad-induced declines, but post-toad numbers in their study remained within the range of highly variable pre-toad counts. Mesopredator releases of Gilbert's dragons and common tree snakes caused by toad-induced predator losses in the shorter-term study (Doody et al. 2009) differed markedly in magnitude and pattern between species (Fig. 3). Gilbert's dragons showed dramatic increases; counts were nearly an order of magnitude greater in the current, medium-term study, while snakes counts were similar between the two studies (short and mediumterms, Fig. 3; see also (Doody et al. 2009, 2013). We hypothesize that increases in both mesopredators were caused by the virtual disappearance of $V$. panoptes from the communities, as the latter preys upon the former (Doody et al. 2012a,b). Importantly, our data do not allow us to distinguish between numerical increases in mesopredator numbers vs. behavioral releases (mesopredators may have foraged more boldly in the absence of $V$. panoptes). Regardless of mechanism, our mesopredator data suggest that, after many years, the relative densities of predators and prey continue to shift.

Despite our diverse but clear results, our study was limited to two sites $\sim 30 \mathrm{~km}$ apart in the same river system; although this distance would seem to provided site independence, predator responses across the range would be required to confirm generality of effects. As for invasive toads as the trigger for observed effects, we are unaware of any other major perturbation in the system during the study period. Wet season flooding of the river is an annual event, and is more extreme in some years, but its timing did not coincide with the timing of changes in species abundances (Australian Bureau of Meteorology).

Although acute (short-term) effects of species invasions can be critical, chronic effects, or the eventual outcomes of invasions, may be of greater ecological and economic importance (Strayer et al. 2006). Acute and chronic effects can yield very different results; red imported fire ants (Solenopsis invicta) have dramatic population-level effects shortly after invasion, but these effects are transient in the longer-term (Porter and Savignano 1990, Morrison 2002), while the acute effects of an invasive reef-building polychaete (Ficopotamus enigmaticus) were minor compared to chronic effects over decades (Schwindt et al. 2001, 2004). Currently, the few studies accurately documenting chronic effects of species invasions are insufficient to provide an empirical foundation (and thus a theoretical framework) for predicting how the ecological effects of an invader will change over time (Strayer et al. 2006). In a review of published works the on the effects of species invasions, only 12 of 199 studies spanned $>10$ yr (Strayer et al. 2006). On the one hand, our study adds to this small list of long-term studies; on the other hand, our study suggests that the system is still in turmoil, having not yet reached a 'new equilibrium' more than a decade after the initial invasion. Given that cane toads have proven too prolific to eradicate or control, we suggest that recovery of impacted predators must occur unassisted via evolutionary means. Evolution and subsequent dispersal may be the only solution for a number of species or communities affected by invasive species for which control is either prohibitively expensive, or not possible. Regardless, by understanding the long-term feedbacks between invasive species and their communities and ecosystems, we will be better placed to evaluate alternative management approaches and predict which future invaders should be targeted because of unacceptable acute or chronic impacts (Strayer et al. 2006).

\section{AcKnowledgments}

We thank R. Allen, G. Brown, L. Crimmins, G. Dryden, L. Grice, D. Hunter, G. Kay, P. Macak, R. Sims, D. Steer, and D. Trembath for assistance in the field and the Douglas-Daly Research Farm for logistical support. We thank M. Letnic for useful discussion. Funding was provided by CSIRO, the Australian Government (Natural Heritage Trust Scheme), and the Jane Fenwick Foundation.

\section{Literature Cited}

Beschta, R. L., and W. J. Ripple. 2013. Are wolves saving Yellowstone's aspen? A landscape-level test of a behaviorally mediated trophic cascade: comment. Ecology 94:1420-1425.

Catling, P. C., A. Hertog, R. J. Burt, J. C. Wombey, and R. I. Forrester. 1999. The short-term effect of cane toads (Bufo marinus) on native fauna in the Gulf Country of the Northern Territory. Wildlife Research 26:161-185.

Cogger, H. 2000. Reptiles and amphibians of Australia. Reed New Holland, Sydney, Australia.

Covacevich, J., and M. Archer. 1975. The distribution of the Cane Toad Bufo marinus in Australia and its effects on indigenous vertebrates. Memoirs of the Queensland Museum 17:305-310.

Doody, J. S., B. Green, R. Sims, D. Rhind, P. West, and D. Steer. 2006. Indirect impacts of invasive cane toads (Bufo marinus) on nest predation in pig-nosed turtles (Carettochelys insculpta). Wildlife Research 33:349-354. 
Doody, J. S., B. Green, D. Rhind, C. M. Castellano, R. Sims, and T. Robinson. 2009. Population-level declines in Australian predators caused by an invasive species. Animal Conservation 12:46-53.

Doody, J. S., M. Hall, D. Rhind, B. Green, and G. Dryden. 2012a. Varanus panoptes (Yellow-spotted Monitor). Diet. Herpetological Review 43:491-492.

Doody, J. S., R. Lloyd, and D. Rhind. 2012b. Varanus panoptes (Yellow-spotted Monitor). Diet and prey capture. Herpetological Review 43:339-340.

Doody, J. S., C. Castellano, D. Rhind, and B. Green. 2013. Indirect facilitation of a native mesopredator by an invasive species: Are cane toads re-shaping tropical riparian communities? Biological Invasions 15:559-568.

Doody, J. S., P. Mayes, S. Clulow, D. Rhind, B. Green, C. Castellano, D'Amore, D. and C. McHenry. 2014. Impacts of the invasive cane toad on aquatic reptiles in a highly modified ecosystem: the importance of replicating impact studies. Biological Invasions 16:2303-2309.

Doody, J. S., R. Soanes, C. M. Castellano, D. Rhind, B. Green, C. McHenry, and S. Clulow. 2015. Invasive toads shift predator-prey densities in animal communities by removing top predators. Ecology 96:2544-2554.

Estes, J. A., J. Terborgh, J. S. Brashares, M. E. Power, J. Berger, W. Bond, et al. 2011. Trophic downgrading of planet earth. Science 333:301-306.

Fukuda, Y., R. Tingley, B. Crase, G. Webb, and K. Saalfeld. 2015. Long-term monitoring reveals declines in an endemic predator following invasion by an exotic species. Animal Conservation 19:75-87.

Griffiths, A. D., and J. L. McKay. 2007. Cane toads reduce the abundance and site occupancy of Merten's water monitor (Varanus mertensi). Wildlife Research 34:609-615.

Letnic, M., and S. Ward. 2005. Observations of freshwater crocodiles (Crocodylus johnstoni) preying upon cane toads (Bufo marinus) in the Northern Territory. Herpetofauna 35:98-100.

Letnic, M., J. K. Webb, and R. Shine. 2008. Invasive cane toads (Bufo marinus) cause mass mortality of freshwater crocodiles (Crocodylus johnstoni) in tropical Australia. Biological Conservation 141:1773-1782.

Lever, C. 2001. The cane toad: the history and ecology of a successful colonist. Westbury Academic and Scientific Publishing, West Yorkshire, UK.

Losos, J. B., and H. W. Green. 1988. Ecological and evolutionary implications of diet in monitor lizards. Biological Journal of the Linnean Society 35:379-407.

Morrison, L. W. 2002. Long-term impacts of an arthropodcommunity invasion by the imported fire ant, Solenopsis invicta. Ecology 83:2337-2345.

Paden, L. 2008. Varanus panoptes horni (argus monitor) sexual maturity. Biawak 2:173-174.

Phillips, B., G. Brown, and R. Shine. 2003. Assessing the potential impact of cane toads on Australian snakes. Conservation Biology 17:1738-1747.

Pianka, E., and D. King. 2004. Varanoid lizards of the world. Indiana University Press, Bloomington, Indiana, USA.

Porter, S. D., and D. A. Savignano. 1990. Invasion of polygyne fire ants decimates native ants and disrupts arthropod community. Ecology 71:2095-2106.

Ripple, W. J., and R. L. Beschta. 2007. Restoring Yellowstone's aspen with wolves. Biological Conservation 138:5147-5519.

Rodriguez, L. F. 2006. Can invasive species facilitate native species? Evidence of how, when, and why these impacts occur. Biological Invasions 8:927-939.

Schwindt, E., A. Bartolus, and O. O. Iribarne. 2001. Invasion of a reef-builder polychaete: direct and indirect effects on the native benthic community structure. Biological Invasions 3:137-149.
Schwindt, E., O. O. Iribarne, and F. I. Isla. 2004. Physical effects of an invasive reef-building polychaete on Argentinian estuarine environment. Estuarone, Coastal and Shelf Science 59:109-120.

Seebacher, F., and G. C. Grigg. 1997. Patterns of body temperature in wild freshwater crocodiles, Crocodylus johnstoni: thermoregulation vs. thermoconformity, seasonal acclimatization, and the effect of social interactions. Copeia 1997:549-557.

Shanmuganathan, T., J. Pallister, S. Doody, H. McCallum, T. Robinson, A. Sheppard, et al. 2010. Biological control of the cane toad in Australia: a review. Animal Conservation 13 (Supplement 1):16-23.

Shine, R. 2010. The ecological impact of invasive cane toads (Bufo marinus) in Australia. Quarterly Review of Biology 85:253-291.

Shine, R., and J. S. Doody. 2011. Invasive species control: understanding conflicts between researchers and the general community. Frontiers in Ecology and the Environment 9:400-406.

Stockwell, C. A., A. P. Hendry, and M. T. Kinnison. 2003. Contemporary evolution meets conservation biology. Trends in Ecology \& Evolution 18:94-101.

Strayer, D. L., E. V. Eviner, J. M. Jeschke, and M. L. Pace. 2006. Understanding the long-term effects of species invasions. Trends in Ecology \& Evolution 21:645-651.

Ujvari, B., and T. Madsen. 2009. Increased mortality of naïve varanid lizards after the invasion of non-native cane toads (Bufo marinus). Herpetological Conservation and Biology 4:248-251.

Ujvari, B., H. Mun, A. Conigrave, A. Bray, J. Osterkamp, P. Halling, et al. 2013. Isolation breeds naivety: Island living robs Australian varanid lizards of toad toxin immunity via four-base-pair mutation. Evolution 67:289-294.

Ujvari, B., N. R. Casewell, K. Sunagar, K. Arbuckle, W. Wuster, N. Lo, D. O’Meally, C. Beckmann, G. King, E. Deplazes, and T. Madsen. 2015. Widespread convergence in toxin resistance by predictable molecular evolution. Proceedings of the National Academy of Sciences of the United States of America 112:11911-11916.

Ward, S. 2012. Threatened species of the Northern territory. Mitchells' water monitor. Varanus mitchelli. Northern Territory Government, Darwin, Australia.

Ward, S., J. Woinarski, T. Griffiths, and L. McKay. 2006. Threatened species of the Northern territory. Mertens water monitor. Varanus mertensi. Northern Territory Government, Darwin, Australia.

Ward, S., J. Woinarski, A. D. Griffiths, and J. L. McKay. 2012. Threatened species of the Northern territory. Yellow-spotted monitor. Varanus panoptes. Northern Territory Government, Darwin, Australia.

Ward-Fear, G., D. J. Pearson, G. P. Brown, B. Rangers, and R. Shine. 2016. Ecological immunization: in situ training of free-ranging predatory lizards reduces their vulnerability to invasive toxic prey. Biology Letters 12:20150863.

Webb, G. J. W., and S. C. Manolis 2010. Australian freshwater crocodile Crocodylus johnstoni. Pages 66-70 in S. C. Manolis, and C. Stevenson, editors. Crocodiles. Status survey and conservation action plan. Crocodile Specialist Group, Darwin, Australia

White, E. M., J. C. Wilson, and A. R. Clarke. 2006. Biotic indirect effects: a neglected concept in Invasion biology. Diversity and Distributions 12:443-455.

Woinarski, J. C. Z., M. Armstrong, K. Brennan, A. Fisher, A. D. Griffiths, B. Hill, et al. 2010. Monitoring indicates raid and severe decline of native small mammals in Kakadu National Park, northern Australia. Wildlife Research, 37:116-126. 